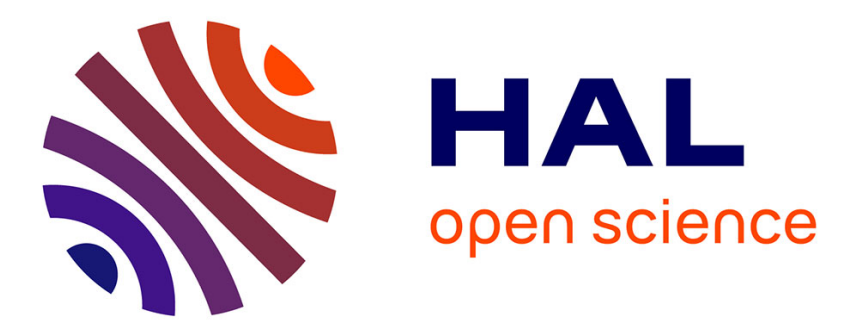

\title{
On the use of discontinuous elastomer patches to optimize the damping properties of composite sandwich plates
}

\author{
Nicolas Le Maoût, Erwan Verron, Jerôme Bègue
}

\section{- To cite this version:}

Nicolas Le Maoût, Erwan Verron, Jerôme Bègue. On the use of discontinuous elastomer patches to optimize the damping properties of composite sandwich plates. Composite Structures, 2011, 93 (11), pp.3057-3062. 10.1016/j.compstruct.2011.04.023 . hal-01007158

\section{HAL Id: hal-01007158 https://hal.science/hal-01007158}

Submitted on 16 Jun 2014

HAL is a multi-disciplinary open access archive for the deposit and dissemination of scientific research documents, whether they are published or not. The documents may come from teaching and research institutions in France or abroad, or from public or private research centers.
L'archive ouverte pluridisciplinaire HAL, est destinée au dépôt et à la diffusion de documents scientifiques de niveau recherche, publiés ou non, émanant des établissements d'enseignement et de recherche français ou étrangers, des laboratoires publics ou privés.

\section{(c)(1)}

Distributed under a Creative Commons Attribution| 4.0 International License 


\title{
On the use of discontinuous elastomer patches to optimize the damping properties of composite sandwich plates
}

\author{
N. Le Maoût ${ }^{\mathrm{a}}$, E. Verron ${ }^{\mathrm{a}}$, J. Bégué ${ }^{\mathrm{b}}$ \\ a Institut de Recherche en Génie Civil et Mécanique, UMR CNRS 6183, Ecole Centrale Nantes, BP 92101, 44321 Nantes cedex 3, France \\ ${ }^{\mathrm{b}}$ Centre Technique des Industries Mécaniques (CETIM), Technocampus EMC2, ZI du Chaffault, 44340 Bouguenais, France
}

This study investigates the optimization of a hybrid composite/elastomer sandwich plate structure using a periodical pattern of viscoelastic material. Design variables consist in the thicknesses of the layers, their fiber orientations, the position of the elastomer patches and their geometrical distribution. Damping properties of the hybrid structure are calculated by the Method of Strain Energy (MSE) and the constrained optimization maximizes these properties using the active-set algorithm. Two optimized plates containing patterns embedded or not in a composite matrix are compared with a plate damped by a continuous viscoelastic layer. Results show that optimizing composite plates with patches gives interesting results: damping properties are about ten times the properties of the plates with continuous viscoelastic layers, the patches can be sticked on the external layers of the plate and not internally, and all the strain components in elastomer are involved in damping.

\author{
Keywords: \\ Passive damping \\ Discontinuous elastomer patches \\ Periodic pattern \\ Method of Strain Energy (MSE) \\ Optimization
}

\section{Introduction}

One important challenge of automotive and aerospace manufacturers is the reduction of noise pollution. Indeed, vibration of structural components is a major source of noise: during vibration, structural parts move, generate air waves and then play the role of a speaker. Due to their low weight and high stiffness, composites are particularly sensitive to this phenomenon. Thus, passive solutions have been proposed to solve this problem: they consist in sticking rubber patches in some well-chosen locations of the structure; they are revealed quiet efficient [1]. Nevertheless, such method must be achieved a posteriori and it modifies the mechanical properties or/and the mass of the structure. To overcome this design difficulty, Kerwin was one of the first authors to consider a sandwich beam including, a priori, internal layers of elastomer [2]. This method has been thoroughly studied by others and has demonstrated its efficiency [3-6]. However, the damping properties of the structure are strongly limited by the loss of stiffness and the increase in mass of the structure induced by the use of elastomer layers.

The majority of studies devoted to sandwich structures damped by rubber layers consider continuous layers of damping material. Recently, Kristensen et al. study a beam damped by a discontinuous viscoelastic layer with gaps filled by composite material [7]. They show that cutting away parts of the damping layer, for example to apply the loads or to place supports, does not permit to significantly reduce the modal loss factor of the structure. In fact, authors underline the importance of using a continuous elastomer layer to obtain relevant damping properties. It is to note that these results have be established only for beam problems with a limited number of design variables.

Even if the conclusions drawn by Kristensen et al. are not so encouraging, we propose here to investigate more deeply the possibility to optimize the damping properties of composite plates by inserting discontinuous elastomer damping layers in sandwich structures. More precisely, we study and compare three different damped plates: the first one with continuous viscoelastic layers, the second one with periodic circular elastomer patches embedded in composite constraining layers and the last one with periodic circular patches surrounded by unfilled gaps. In order to determine the best distribution of elastomer patterns, the numerical tool presented in [8] is used, and several design variables, i.e., thicknesses and positions of patches, are simultaneously optimized.

\section{Optimization of sandwich plates with elastomer patches}

\subsection{Geometry and materials}

Sandwich plates are rectangular and the viscoelastic patches are embedded in constraining layers or glued to the external layers of the structure. The position of patches, their size and distribution are considered as design variables of the optimization problem. Two different hybrid plates with discontinuous layers are studied: 


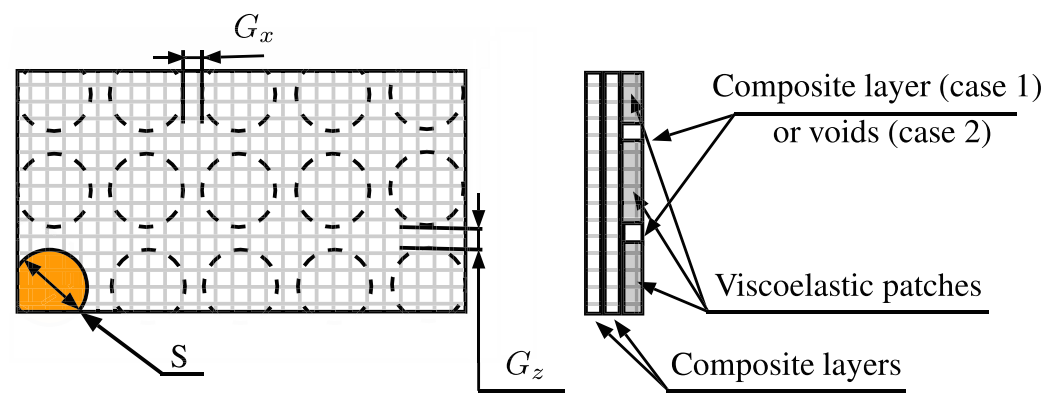

Fig. 1. Geometry of damping layers: distribution of the circular patterns and geometrical data (face and profile views).

- first, a plate with patches embedded in composite layers is considered. In this case, elastomer patches are surrounded by gaps filled with composite material,

- second, a plate with patches glued to composite layers is studied. In this case, the elastomer layers contain unfilled gaps between the patches.

In the following the former structure is referred to as plate (or case) 1 and the latter as plate (or case) 2. The geometry of the bi-material layers is presented in Fig. 1. We choose to adopt circular patches which diameter is denoted $S$. The gaps between patches are denoted $G_{x}$ and $G_{z}$ in the $x$ - and $z$-directions respectively. Obviously, the number of patches in the layer is determined by these parameters These optimized plates are compared with a $200 \times 125 \mathrm{~mm}^{2}$ 3-layer plate that includes continuous viscoelastic layers. Its stacking sequence and the thicknesses of layers have been optimized in [8] and will be presented in the Section 3 .

Remark 1. For the sake of simplicity, we only consider circular patches. Indeed, the aim of this study is to demonstrate the relevance of using patches to damp composite plates and not to discuss their possible geometry. More precisely, circular patches are representative of all regular polygons without the complexity of their orientation, so this choice minimizes the number of geometrical characteristics of the patches.

For the three plates, the material of composite layers is a glass fiber-reinforced epoxy which parameters are issued from Berthelot et al. [9]: the material is orthotropic and, denoting 1 the fiber direction and 2, 3 the transverse directions, the Young moduli are $E_{1}=$ $29.9 \mathrm{GPa}, E_{2}=E_{3}=7.5 \mathrm{GPa}$, the shear modulus is $G_{12}=2.25 \mathrm{GPa}$ and the Poisson ratios are $v_{12}=v_{23}=v_{13}=0.24$. Its density is equal to $1500 \mathrm{~kg} \mathrm{~m}^{-3}$. The rubber material used for the patches is assumed isotropic and linearly viscoelastic; its Young modulus $E(f)$ is considered frequency $(f)$-dependent and the expression issued from Zhang and Chen [10] is retained:

$$
E(f)=0.0041+0.0322 \log (f) \mathrm{MPa}
$$

The Poisson ratio is constant $v=0.3$ and the density is $968.1 \mathrm{~kg} \mathrm{~m}^{-3}$. Material loss factor $\eta_{v}$ is chosen real, constant and set equal to 0.3 . Finally, for the plate with unfilled gaps, air is modeled with a very low density and mechanical properties: $1.1 \mathrm{~kg} \mathrm{~m}^{-3}, E=0.1 \mathrm{MPa}$ and $v=0.3$. For more details on the tensorial forms of the constitutive equations and their numerical implementation, the reader can refer to [8].

\subsection{Optimization conditions}

The damping properties of a given structure are determined with classical free vibrations computations between $1 \mathrm{~Hz}$ and $1000 \mathrm{~Hz}$, considering free-displacement boundary conditions on the plate edges. By using the Method of Strain Energy (MSE in the following) [11], this calculation permits to determine the modal loss factors $\eta_{i}$ of the plates for each modal frequency $i$ in the range $[1 \mathrm{~Hz}, 1000 \mathrm{~Hz}]$. More precisely, the $i^{\text {est }}$ modal loss factor for which the eigenfrequency is $f_{i}$ is

$\eta_{i}=\eta_{v} \frac{W}{W_{\text {tot }}}$

where $W$ is the strain energy in the elastomer material and $W_{\text {tot }}$ is the total strain energy in the structure. Optimization of the damping properties necessitates the definition of an objective function $\mathscr{F}$ that includes the modal loss factors in the range [ $1 \mathrm{~Hz}, 1000 \mathrm{~Hz}$ ]; here we simply consider the reciprocal average of the loss factors

$\left.\mathscr{F}=\frac{1}{N} \sum_{i=1}^{N} \eta_{i}\right)^{-1}$

in which $N$ is the number of eigenfrequencies in the frequency range.

Remark 2. The choice of this objective function is motivated by its simplicity: it permits to easily encompass the whole range of frequencies. Obviously, defining functions that emphasize some given responses of the structure, e.g., high frequencies, only takes the addition of well-chosen weighting functions in $\mathscr{F}$.

Nevertheless, the comparison of damping capacities of the plates is relevant only if these plates admit comparable mass and mechanical properties. In this way, constraints are added to the optimization problem. The mass constraint is obvious: the increase in mass due to the addition of viscoelastic layers is limited. For the mechanical properties, we consider two different mechanical tests as shown in Fig. 2. First, tensile stiffnesses are estimated by two uniaxial extension tests in both $x$ and $z$-directions as shown in Fig. 2a: displacements $\Delta d=1 \mathrm{~mm}$ are prescribed and the corresponding reaction forces Ftx and Ftz are recorded. Similarly, a 3-point bending test shown in Fig. 2b is considered: the central punch prescribes a $2 \mathrm{~mm}$ deflection in the center of the plate and stiffness is estimated through the reaction force $F b$. Then, minimization of $\mathscr{F}$ is constrained by considering that the differences in mass and tensile stiffness between the structure with viscoelastic patches and the reference structure, i.e., the one with continuous viscoelastic layers, must not exceed $1.5 \%$.

The design variables that are optimized and the corresponding bounds are:

- The number of layer: $n$,

- The nature of each layer: $V_{i=1 \ldots n} . V_{i}<0.5$ : patch layer, $V_{i} \geqslant 0.5$ : composite layer,

- The fiber orientation in each layer: $0^{\circ} \leqslant \theta_{i=1 \ldots n} \leqslant 90^{\circ}$,

- The thickness of each layer: $0.1 \mathrm{~mm} \leqslant t_{i=1 \ldots n} \leqslant 3 \mathrm{~mm}$,

- The diameter of patches: $5 \mathrm{~mm} \leqslant S \leqslant 250 \mathrm{~mm}$, 


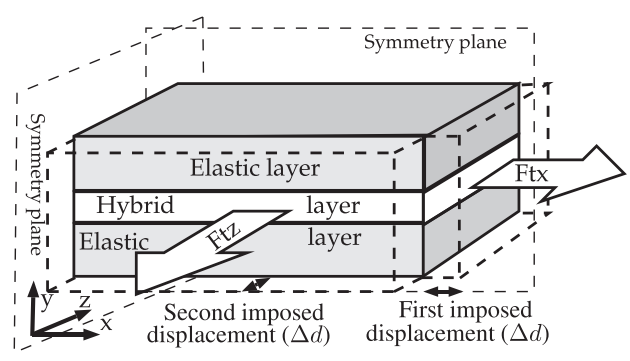

(a)

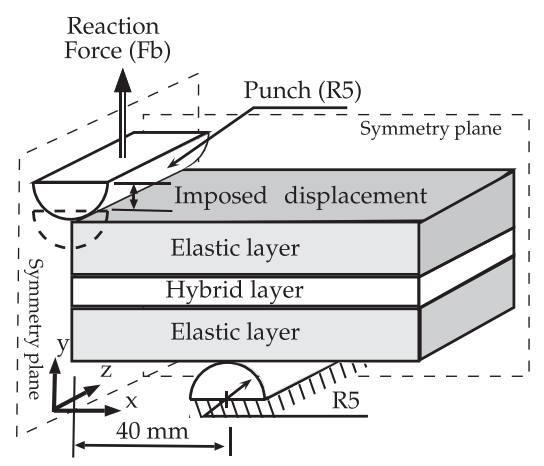

(b)

Fig. 2. (a) Uniaxial tensile tests. (b) Three-point bending test.

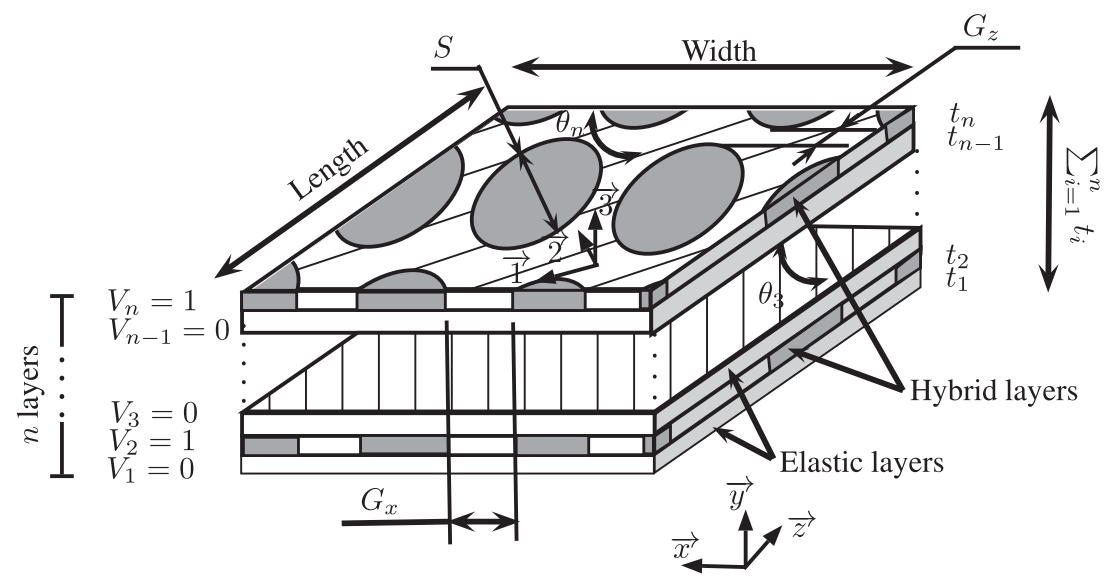

Fig. 3. Geometry of the sandwich plate and design variables.
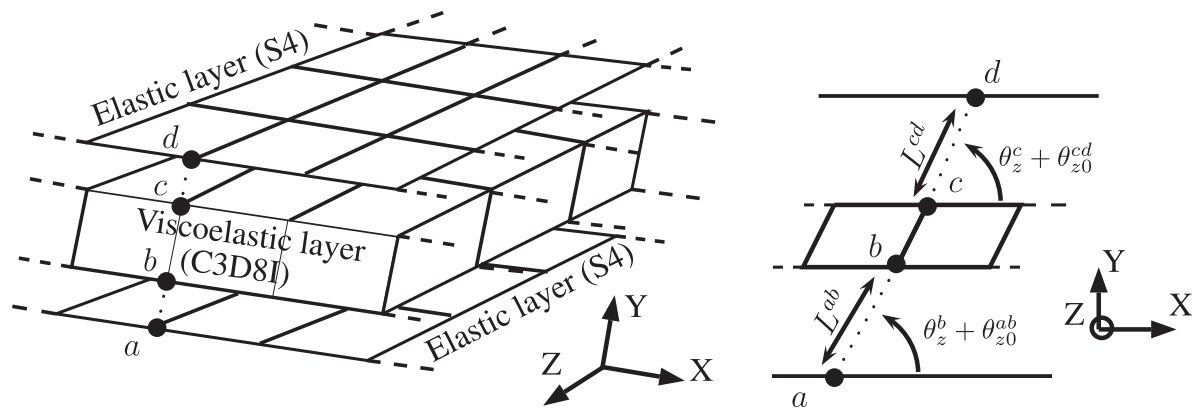

Fig. 4. 3-layer sandwich: mesh of the structure (left-hand side) and coupling between nodes (right-hand side).

- The gaps between patches along $x$ - and $z$-directions: $1.1 \mathrm{~mm} \leqslant$ $G_{x} \leqslant 125 \mathrm{~mm}$ and $1.1 \mathrm{~mm} \leqslant G_{z} \leqslant 125 \mathrm{~mm}$.

The geometry of the sandwich plate and the design variables are presented in Fig. 3.

\subsection{Finite element model}

Here, only new informations are given: loading conditions, numerical methods and optimization features are detailed in [8].

It is recognized that damping layers dissipate energy through transverse shear deformation (see for example [2]), thus hybrid layers are meshed with linear brick elements C3D8I (Abaqus name) with incompatible modes (to improve the bending response of the whole mesh). Moreover, in order to reduce the computing time, we adopt some assumptions for the composite layers to reduce the number of degrees of freedom of the model: (i) shear in the thickness direction of the composite layers is negligible as compared to other stress and strain components, (ii) the transverse displacements are continuous between two neighboring layers, and finally (iii) adhesion between layers is perfect. With these three assumptions, it is possible to mesh the composite layers with 4-node shell elements. Obviously, the coupling between elastomer solid elements and composite shell elements necessitate additional constraints. These constraints are schematized in Fig. 4; they relate the displacement and rotation at a node of the shell, i.e., in the 
mid-plane of the composite layer, to the displacement of the corresponding node of the damping layer. It is achieved by considering rigid truss connectors between nodes, i.e., between nodes a and b, and $\mathrm{c}$ and $\mathrm{d}$ in Fig. 4. Even with this choice, the number of degrees of freedom remains large. So, to limit computing time, we have preliminarily determined an optimal mesh size by considering a 3-layer cantilever beam with isotropic materials as presented in [8]. This structure was chosen because it has been thoroughly studied theoretically, numerically and experimentally [12-14]. The inplane optimal mesh size is equal to $0.55 \mathrm{~mm}$ and we proved that one element by half millimeter in the thickness direction of the viscoelastic layer is sufficient to capture phenomena.

As mentioned above, the viscoelastic layers are meshed with brick elements. Nevertheless, the heterogeneous nature of these layers (patches and air in plate 1, or patches and composite in plate 2 ) necessitates some particular methods to be able to modify the geometry of the patches throughout the optimization loop: we consider a regular mesh and the patch geometry evolves by changing the mechanical properties of the relevant finite elements. In order to determine the elements which properties must changed, the Matlab "inpolygon" function is used: once the position of a circular patch is determined, all the elements which centers are contained inside the polygonal region defined by the circle equation are selected. The starting point of the pattern is obtained by considering an initial patch in the center of the layer; then the pattern is generated by considering a linear distribution of patches defined by the gap parameters $G_{x}$ and $G_{z}$.

Finally, the linear-search algorithm of Matlab is used to solve the constrained optimization problem; it is associated with the finite element code Abaqus (see details in [8]).

\section{Results and discussion}

The aim of this study is to evaluate the relevance of hybrid/heterogeneous viscoelastic layers to damp sandwich plates. In order to reduce computing time but also to simplify the subsequent discussion, the number of layers is fixed to $n=3$, including the patched layer. Thus, recalling the list of design variables given in Section 2.1, 12 variables are simultaneously optimized.

The results of the optimization procedure are presented in Table 1 . The design variables are given for both plates (with unfilled and filled gaps) in the third and fourth columns respectively, and compared to the reference structure which data are given in the second column. Nonlinear constraints are respected at the end of the optimization: in both cases, with filled and void gaps, decrease in tensile stiffness and increase of weight do not exceed $1.5 \%$. It is to note that the bending stiffness $F b$ is highly improved as discontinuous elastomer layers replace continuous layers and that this reinforcement is larger for void gaps between patches.

The primary result concerns the layers sequence: for the reference plate, the optimal sequence consists in placing the viscoelas-

Table 1

Design parameters and constraints issued from the optimization of the 3-layer sandwich plate. "V" denotes the position of the continuous viscoelastic layer, and " $\mathrm{HX}$ " denotes the position of the hybrid layer and the fiber orientation of the matrix $X$.

\begin{tabular}{llll}
\hline & 3-layer (reference) & Plate 1 (filled gaps) & Plate 2 (void gaps) \\
\hline$\theta\left(^{\circ}\right)$ & {$[0 / \mathrm{V} / 90]$} & {$[\mathrm{HO} / 0 / 90]$} & {$[\mathrm{H}-/ 0 / 90]$} \\
$t(\mathrm{~mm})$ & {$[0.57 / 0.34 / 0.47]$} & {$[0.63 / 0.30 / 0.46]$} & {$[0.59 / 0.56 / 0.46]$} \\
$S(\mathrm{~mm})$ & - & 76.51 & 25.64 \\
$G_{x / z}(\mathrm{~mm})$ & - & $4 / 20.36$ & $4.42 / 3$ \\
$M(\mathrm{~kg})$ & 0.0472 & $0.04789(+1.48 \%)$ & $0.04749(+0.6 \%)$ \\
$F b(\mathrm{~N})$ & -42.0 & $-74.0(+76 \%)$ & $-98(+133 \%)$ \\
$F t x(\mathrm{kN})$ & 14.334 & $14.190(-1 \%)$ & $14.119(-1.5 \%)$ \\
$F t z(\mathrm{kN})$ & 12.712 & $12.645(-0.53 \%)$ & $12.521(-1.5 \%)$ \\
& Nb iterations & 252 & 184 \\
& CPU time $(\mathrm{h})$ & 40 & 30 \\
\hline
\end{tabular}

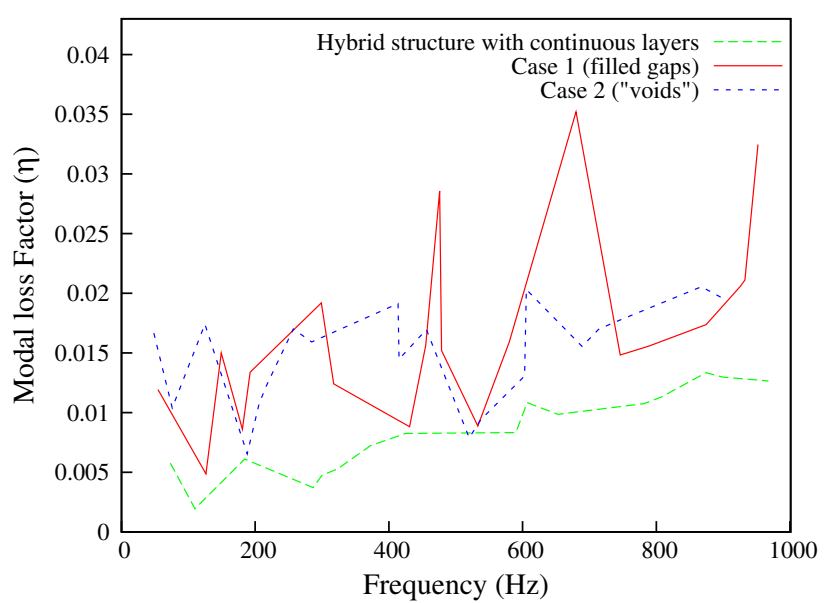

Fig. 5. Evolution of the modal loss factor in the frequency range $[1 \mathrm{~Hz}, 1000 \mathrm{~Hz}]$ for the three configurations studied.

tic layer between the composite layers whereas the optimal patched plates consist in sticking the viscoelastic layers (patches with or without composite) on one of the extreme layers. From a manufacturing point of view, this result is of major importance because sticking patches on a sandwich plate is highly easier than inserting viscoelastic layers during forming. Obviously, this results must be analyzed by also examining the thicknesses of the layers; to obtain similar damping performances, plate 1 (with filled patches) is approximately as thick as the reference plate (1.39 mm vs. $1.38 \mathrm{~mm}$ ) whereas plate 2 (with void gaps) must be thicker than the reference plate $(1.64 \mathrm{~mm})$.

Second, we examine the geometry of the patched layers. For plate 1, i.e., the plate in which the gaps are filled with composite, the sum of the pattern size $S$ and $z$-gap $G_{z}$ is of the same order of magnitude than the dimension of the plate $(76.51+2 \times 20.36=$ $117 \mathrm{~mm} \approx 125 \mathrm{~mm}$ ); thus, only one patch is necessary along the smallest edge. The longer distance between the patches along the other edge, $G_{x}$ stiffens the whole structure during static tests. The optimization of plate 2 that contains patches surrounded by voids gives a very different geometrical distribution: the diameter of the patches is smaller than in the previous case and gaps between patches along both directions leads to a layer with numerous patches. To illustrate this results, the reader can examine the Fig. 6 in which the position of the patches corresponds to the blue ${ }^{1}$ circles (dark circles for gray scale pictures).

The evolution of the modal loss factors in the frequency range studied is shown in Fig. 5. It shows that plate 1 has globally the best damping properties. First, as compared to the reference plate with a continuous layer, the difference between loss factors can exceed $100 \%$ for some eigenfrequencies. Second, the difference with plate 2 (with void gaps) is not so important: depending on the eigenfrequency considered, one or the other configurations admits the best damping properties. Roughly, the response of plate 2 is more regular as a function of the frequency, and seems to be well-adapted to small frequencies (less than $150 \mathrm{~Hz}$ ), and plate 1 is revealed highly more performing for some high frequencies (between 600 and $700 \mathrm{~Hz}$ ) for example. Moreover, it is to note that both solutions with patches admit more eigenfrequencies than the reference plate. In Fig. 6, we present an example of the strain energy distribution in deformed plates; the pictures correspond to the 7th and 8th modes for filled and void patches, respectively. To simplify the visualization of plate 2 , finite elements for the air

\footnotetext{
${ }^{1}$ For interpretation of color in Fig. 6, the reader is referred to the web version of this article.
} 

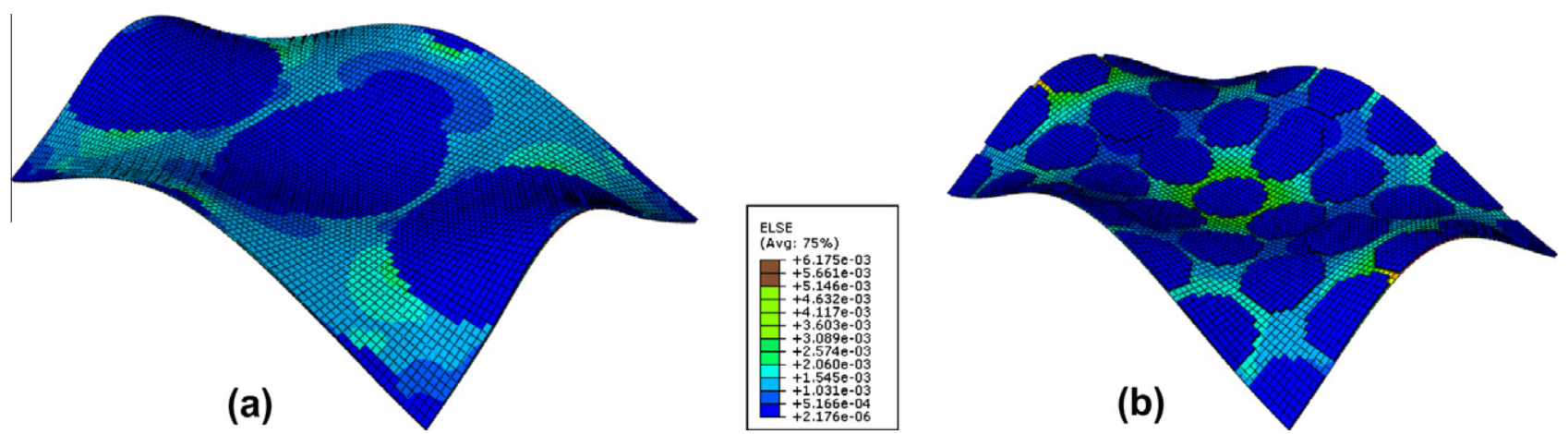

Fig. 6. Strain energy distribution in deformed plates: (a) plate 1 for the 7 th eigenfrequency $(f=318 \mathrm{~Hz})$, (b) plate 2 for the 8 th eigenfrequency $(f=285 \mathrm{~Hz})$.

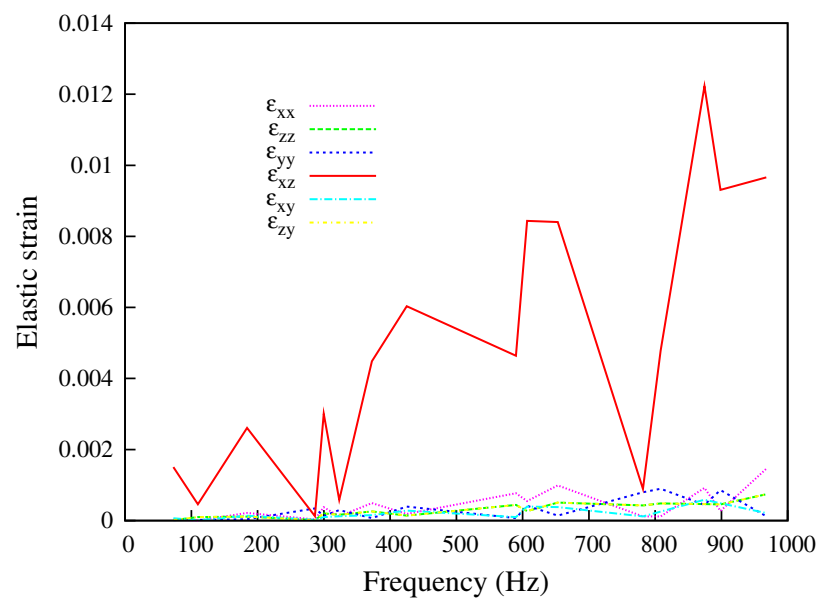

(a)

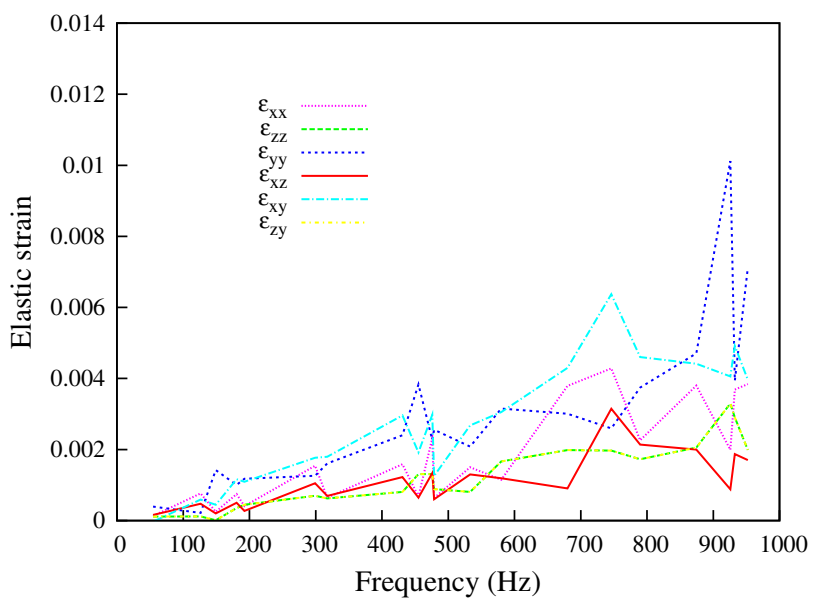

(b)

Fig. 7. Maximum strain components: (a) in the reference structure, (b) in plate 1, with patches surrounded by filled gaps.

which surround the patches have been deleted. As both pictures are drawn with the same energy scale, we can conclude that the energy in the plates are comparable in elastomer but also in the composite (in composite that surrounds patches for plate 1 and in the composite layer under the patches for plate 2); this can be also verified by noting that the modal loss factor of the two plates are of the same order of magnitude for frequencies of about $300 \%$ (see Fig. 5).

Fig. 7 shows the maximum values of the strain tensor components in the viscoelastic layer as a function of the frequency; it compares the response of the plate with filled gaps (plate 2, Fig. 7b) with the reference plate (Fig. 7a). For the reference plate, we recover the classical result: the major mechanism for damping in sandwich plates with viscoelastic layers is the shearing of the viscoelastic layer as shown by the predominance of $\varepsilon_{x z}$. In contrast, for the plate with patches, all the strain components are almost equivalently involved in damping; it explains the better performance of the patched plate for similar thicknesses.

\section{Conclusion}

In this paper, the numerical tool presented in [8] has been used to maximize the passive damping capabilities of multi-layer plates with respect to a large number of variables. Optimization has been performed with linear and non-linear constraints to limit the increase in mass and the decrease in stiffness of the hybrid structure as compared to the undamped one. Here, hybrid structures con- taining elastomer patches were investigated. Two types of structure were considered, optimized and compared to a three-layer reference plate made of two composite layers separated by a continuous viscoelastic layer: the first plate contains a hybrid layer of elastomer patches surrounded by the composite material, and the second one contains a layer of patches surrounded by voids.

As mentioned in the introduction, Kristensen et al. recently argue that such solution does not give interesting results [7]. Here, we demonstrate that the use of viscoelastic patches is very efficient if the sandwich structure is globally optimized a priori: composite properties (positions, orientations and thicknesses of the layers) and patches properties (thickness, size and position of the patches) must be determined simultaneously. The major results are:

- whereas optimized plates with continuous viscoelastic layers consist in placing the elastomer layer between the composite layers, optimized plates with patches consist in gluing the elastomer patches on external composite layers for both patches embedded in composite or not,

- even if the number of eigenfrequencies is significantly increased, damping properties of patched plates are about ten times greater than for classical damped plates,

- this result is mainly due to the fact that all strain components are involved in damping,

- finally, the total thickness of the plates is preserved for patches surrounded by composite, but increased (of about 25\% in the problem studied here) for the plate with patches surrounded by unfilled gaps. 
To close this paper, we claim that the passive solution that consists in patches glued on composite plates is a good alternative to introducing viscoelastic layers in sandwich plates, especially in terms of manufacturing because the patches can be deposited $a$ posteriori on the plate surface. Nevertheless, such solution is relevant only if the structure is optimized a priori. Further investigations are now necessary to confirm these preliminary results and also to optimize the geometrical characteristics of the patches.

\section{References}

[1] Zheng H, Cai C, Tan XM. Optimization of partial constrained layer damping treatment for vibrational energy minimization of vibrating beams. Comput Struct 2004;82:2493-507.

[2] Kerwin EM. Damping of flexural waves by a constrained viscoelastic layer. J Acoust Soc Am 1959;37(7):952-62.

[3] Rao DK. Frequency and loss factor of sandwich beams under various boundary conditions. J Mech Eng Sci 1978;20(5):271-82.

[4] Lu YP, Killian JW, Everstine GC. Vibrations of three layered damped sandwich plate composites. J Sound Vib 1979;64(1):63-71.
[5] Chen X, Chen HL, Hu XL. Damping predication of sandwich structures by orderreduction-iteration approach. J Sound Vib 1999;222(5):803-12.

[6] Kishi H, Kuwata M, Matsuda S, Toshihiko A, Atsushi M. Damping properties of thermoplastic-elastomer interleaved carbon fiber-reinforced epoxy composites. Compos Sci Technol 2004;64:2517-23.

[7] Kristensen RF, Nielsen KL, Mikkelsen LP. Numerical studies of shear damped composites beams using a constrained damping layer. Compos Struct 2008;83:304-11.

[8] Le Maoût N, Verron E, Bégué J. Simultaneous geometrical and material optimal design of hybrid elastomer/composite sandwich plates. Compos Struct 2011;93:1153-7.

[9] Berthelot J-M, Assarar M, Sefrani Y, El Mahi A. Damping analysis of composite materials and structures. Compos Struct 2008;85:189-204.

[10] Zhang SH, Chen HL. A study on the damping characteristics of laminated composites with integral viscoelastic layers. Compos Struct 2006;74:63-9.

[11] Ungar EE, Kerwin Jr EM. Loss factors of viscoelastic systems in terms of strain energy. J Acoust Soc Am 1962;34(2):954-62.

[12] Soni ML. Finite element analysis of viscoelastically damped sandwich structures. Shock Vib Bull 1981:55(1):97-109.

[13] Daya EM, Potier-Ferry M. A numerical method for nonlinear eigenvalue problems application to vibrations of viscoelastic structures. Comput Struct 2001;79:533-41.

[14] Johnson CD, Kienholz DA, Rogers LC. Finite element prediction of damping in beams with constrained viscoelastic layers. Shock Vib Bull 1981;51(1):71-81. 